\title{
Nutrição hiperproteica em recém-nascidos com muito baixo peso e evolução de índices antropométricos até a alta hospitalar: ensaio clínico randomizado
}

\author{
Eduardo M. Soares ${ }^{1, *}$
}

\begin{abstract}
Resumo
Objetivo: avaliar se o maior aporte proteico enteral durante a internação hospitalar promove melhora dos índices antropométricos na alta hospitalar.

Método: ensaio clínico randomizado com 117 prematuros nascidos entre janeiro de 2009 e julho de 2013 com peso $\leq$ 1500 gramas e idade gestacional $\leq 32$ semanas em uma unidade terciária de saúde, excluídos os nascidos com malformações graves, aferindo-se os índices antropométricos ao nascimento e na alta hospitalar. Randomizaram-se os prematuros em dois grupos: grupo $1(\mathrm{n}=53)$ submetido a um aporte proteicoenteral diário de 4,5 gramas/kg/dia e grupo 2 (n=64) 3,5 gramas $/ \mathrm{kg} / \mathrm{dia}$. Resultados: verificou-se diferença estatisticamente significativa para retorno ao peso de nascimento $(\mathrm{p}=0,02)$, crescimento de escore- $Z$ em relação ao peso de nascimento $(p=0,03)$ e crescimento escore- $Z$ em relação ao comprimento de nascimento ( $\mathrm{p}=0,02$ ) quando comparados o grupo 1 ao 2 . Não houve diferenças estatisticamente significativas nas incidências de enterocolite necrotizante ( $\mathrm{p}=0,70, \mathrm{OR} 0,88$ ), deficit ponderal na alta ( $\mathrm{p}=0,27$, OR 0,70 ), restrição de crescimento na alta $(\mathrm{p}=0,39$, OR 0,82 ) e deficit de perímetro cefálico na alta ( $\mathrm{p}=0,45$, OR 0,67 ). Conclusão: os participantes do grupo 1 apresentaram menor decréscimo de escores- $Z$ em relação ao peso de nascimento e ao comprimento de nascimento quando comparados ao grupo 2, além de necessidade de menor tempo para recuperação do peso de nascimento. Não houve diferença entre os grupos para tempo de internação hospitalar, assim como para enterocolite necrotizante, deficit ponderal na alta, restrição de crescimento na alta e deficit de perímetro cefálico na alta.
\end{abstract}

Descritores: Prematuridade; Recém-nascido com muito baixo peso; Nutrição hiperproteica; Nutrição agressiva; Crescimento extrauterino restrito; Baixa estatura.

\section{Abstract \\ Hyperproteic nutrition in very low birth weight newborn and evolution of anthropometric indices until hospital discharge: a randomized clinical trial}

Objective: to assess whether the higher enteral protein intake during hospitalization promotes improvement of anthropometric indices in hospital discharge.

Method: randomized clinical trial of 117 preterm infants born between January 2009 and July 2013 with weight $\leq 1500$ grams and gestational age $\leq 32$ weeks in a tertiary health care unit, excluding those born with serious defects, gauging their anthropometric measures at birth and at discharge. The prema-
1. Departamento de Pediatria e Puericultura. Faculdade de Ciências Médicas. Universidade do Estado do Rio de Janeiro. Rio de Janeiro, RJ, Brasil.

*Endereço para correspondência: Rua Pereira Nunes, 350, apto 101B Rio de Janeiro, RJ. CEP: 20541-024.

E-mail: edumacsoares@gmail.com

Revista HUPE, Rio de Janeiro, 2016;15(2):92-98 doi: $10.12957 /$ rhupe.2016.28232 Recebido em 29/06/2016. Aprovado em 14/07/2016.

tures were randomized the preterms drawing into two groups: group $1(n=53)$ was subjected to a daily enteral protein intake of 4.5 grams of protein $/ \mathrm{kg} /$ day and group $2(n=64)$ received 3.5 grams of protein $/ \mathrm{kg} /$ day.

Results: it was verified that there was a statistically significant difference for return to birth weight $(p=0.02)$, Z-score variation in relation to birth weight $(\mathrm{p}=0.03)$ and $\mathrm{Z}$-score variation over the length of birth $(\mathrm{p}=0.02)$ when comparing groups 1 and 2 . There were no statistically significant differences in the incidence of necrotizing enterocolitis ( $p=0.70$, OR 0.88), weight deficit at discharge $(\mathrm{p}=027, \mathrm{RR} 0.70)$, growth restriction at discharge ( $p=0.39$, OR 0.82$)$ and deficit of head circumference at discharge ( $\mathrm{p}=0.45$, OR 0.67$)$.

Conclusion: the participants in group 1 had a lower decrease of Z-scores in relation to birth weight and length of birth when compared to group 2, and need less time to recover birth weight. There was no difference between groups for hospitalization time, as well as for necrotizing enterocolitis, underweight high, growth restriction at discharge and deficit ofcephalic circumference at discharge.

Keywords: Prematurity; Very low birth weight; Hyperproteic nutrition; Aggressive Nutrition; Extrauterine restricted growth; Stunting.

\section{Resumen}

Nutrición hiperprotéica en recién nacidos con muy bajo peso y evolución de los índices antropométricos al alta hospitalaria: ensayo controlado aleatorizado

Objetivo: evaluar si el mayor aporte proteico enteral durante la hospitalización promueve la mejora de los índices antropométricos en el alta hospitalaria. Método: ensayo clínico aleatorizado con 117 bebés prematuros nacidos entre enero de 2009 y julio de 2013 , con peso $\leq 1500$ gramos y edad gestacional $\leq 32$ 
semanas en una unidad de cuidados terciarios, con exclusión de los nacidos con malformaciones graves, comparándose los indices antropométricos al nacer y al alta hospitalaria. Se dividieron aleatoriamente los prematuros en dos grupos: grupo $1(n=53)$ se sometió a un aporte proteicoenteral diario de 4,5 gramos /kg/día y el grupo $2(\mathrm{n}=64) 3,5$ gramos/kg/día. Resultados: se verificó diferencias estadísticamente significativas para volver al peso de nacimiento $(p=0,02)$, crecimiento de Z-score en relación con el peso al nacer $(p=0,03)$ y crecimiento de Z-score en relación con la longitud de nacimiento $(\mathrm{p}=$ 0,02 ) cuando se comparó el grupo 1 al 2 . No hubo diferencias estadísticamente significativas en la incidencia de enterocolitis necrotizante $(\mathrm{p}=0,70$; OR 0,88$)$, déficit ponderal al alta ( $\mathrm{p}=0,27$, OR 0,70 ), restricción del crecimiento al momento

Palabras clave: Prematuridad; Los recién nacidos con muy bajo peso;La nutrición dealto valor proteico;La nutrición agresiva; Restringido el crecimiento extrauterino; La baja estatura.

\section{Introdução}

É crescente a preocupação dos neonatologistas em promover melhorias na nutrição do prematuro, principalmente, durante o período de internação hospitalar. Cogita-se que a desnutrição intra-hospitalar pode apresentar consequências irreversíveis a essa população., ${ }^{1,2}$ Apesar dos diversos estudos realizados nas últimas décadas, ainda não há consenso no modo de alimentação desses pacientes no ambiente intra-hospitalar ${ }^{3,4}$ e, com as atuais estratégias de suporte nutricional, o crescimento do recém-nascido prematuro fica muito aquém do preconizado, ${ }^{5}$ estabelecendo-se o chamado de crescimento extrauterino restrito (CEUR). ${ }^{3}$ É inegável que, mesmo com todos os avanços tecnológicos, os recém-nascidos com muito baixo peso (RNMBP) ainda apresentam deficiência de peso, estatura e perímetro cefálico no momento da alta hospitalar. Carlson e Ziegler ${ }^{6}$ propuseram que o CEUR é consequência do baixo aporte protéico oferecido a esses prematuros, tanto através de nutrição enteral quanto parenteral, pois no ambiente intraútero o aporte básico estimado situa-se entre 4,0 a 4,5 gramas de proteína por quilograma de peso por dia (g/kg/dia).6-8 Embleton $^{9}$ demonstrou que com as atuais estratégias de nutrição, o recém-nascido prematuro com idade gestacional menor que 30 semanas apresenta, ao fim de 5 semanas de internação hospitalar, uma deficiência total aproximada de $23 \mathrm{~g}$ de peso de proteína.

Apesar de demonstrada a necessidade de maior aporte proteico aos prematuros, ainda existe grande temor em relação à oferta hiperproteica enteral a esses del alta $(p=0,39 ;$ OR 0,82$)$ y el déficit de la circunferencia de la cabeza al momento del alta ( $p=0,45 ;$ OR 0,67). Conclusión: los participantes del grupo 1 en comparación con el grupo 2 tuvieron una disminución menor de escores- $\mathrm{Z}$ en relación con el peso y la longitud al nacer, y necesitaron menos tiempo para recuperar el peso del nacimiento. No hubo diferencias entre los grupos en el tiempo de internación hospitalaria, ni en la enterocolitis necrotizante, ni el deficit ponderal en el alta, ni en la restricción del crecimiento en el alta, ni en la circunferencia de la cabeza en el alta.

Palabras clave: Prematuridad; Recién nacidos con muy bajo peso; Nutrición hiperproteica; Nutrición agresiva; crecimiento extrauterino restringido; baja estatura.

pacientes, especialmente devido ao estudo de Goldman, ${ }^{10}$ que demonstrou que prematuros alimentados com maior aporte proteico por via exclusiva enteral com fórmulas apresentavam acidose metabólica, concentrações plasmáticas excessivas de amônia, uremia, hiperaminoacidemia, restrição do crescimento e piora no desenvolvimento. Outra grande barreira ao estabelecimento do emprego rotineiro da nutrição hiperproteica é o temor de que, ao aumentar o aporte proteico-calórico do prematuro, acelerar-se-ia o ganho de peso deste, predispondo-o à síndrome metabólica futura. ${ }^{11,12}$

O presente estudo tem por objetivo avaliar se o maior aporte proteico enteral oferecido a RNMBP durante a internação hospitalar em UTI neonatal promove melhora dos índices antropométricos até a alta hospitalar dos prematuros.

\section{Metodologia}

Ensaio clínico randomizado em dois grupos paralelos, realizado em unidade terciária de saúde de janeiro de 2009 a julho de 2013, sendo estudadas crianças nascidas na unidade classificadas como RNMBP e com idade gestacional $\leq 32$ semanas,excluindo-se aquelas com malformações congênitas ou sequelas graves (cardíacas, gastrointestinais, pulmonares, genéticas e de tubo neural).

A tabela 1 resume o protocolo de nutrição dos participantes do estudo.

A pesagem foi realizada com participantes desnudos, em balança modelo BP Baby (Filizola ${ }^{\circledR}$, São Paulo, SP, Brasil), o comprimento aferido com infantômetro com haste de alumínio (FANEM ${ }^{\circledR}$, São Paulo, SP, Brasil) e o perímetro cefálico com fita inelástica (Macrolife ${ }^{\circledR}$, Pato Branco, PR, Brasil). ${ }^{13}$ 


\section{Artigo original}

Para a classificação dos recém-nascidos do estudo quanto à idade gestacional, utilizou-se como referência a data da última menstruação(DUM); quando indisponível, utilizou-se a ultrassonografia (USG) de primeiro trimestre ou o New Ballard Score. ${ }^{14}$ Os gráficos de crescimento de Fenton e Kim ${ }^{15}$ foram empregados na avaliação de adequação de peso (P/I), do comprimento (E/I) e do perímetro cefálico (PC/I) para idade gestacional.

A classificação dos recém-nascidos quanto à adequação de peso para idade gestacional foi de pequeno para idade gestacional (PIG) quando o escore-Z foi $\leq$ $-2,0$, e grande para a idade gestacional (GIG) quando o escore-Z foi $\geq 2,0$.

$A$ variação de escore- $Z$ da alta em relação ao nascimento para peso (crescimento de escore- $Z \mathrm{PN}$ ), comprimento (crescimento de escore- $\mathrm{Z} \mathrm{CN}$ ) e perímetro cefálico (crescimento de escore-Z PCN) foram calculados através da subtração dos escores- $Z$ de alta de peso, comprimento e perímetro cefálico com seus respectivos escores- $Z$ de nascimento.

Enterocolite necrotizante (NEC) foi considerada quando o paciente apresentou simultaneamente distensão abdominal e telerradiografia de abdome alterada, correspondendo aos graus II e III de Bell. ${ }^{16}$

Deficit ponderal na alta hospitalar (deficit ponderal alta), restrição de crescimento na alta hospitalar (restrição crescimento alta) e deficit de perímetro cefálico na alta (deficit PC alta) foram considerados quando o paciente não alcançava no momento da alta hospitalar escores-Z <-2,0 para P/I, E/I e PC/I respectivamente.

Os dados foram, então, armazenados em planilha montada no software de estatística EPI-INFO versão 3.5.3 (CDC, Atlanta, GA, USA), com o qual se realizaram as análises estatísticas de interesse.

Os cálculos estatísticos realizados foram o teste exato de Ficher e/ou odds relativo (OR) para variáveis categóricas, e ANOVA ou Teste de Kruskal-Wallis para variáveis contínuas. Foi considerado o valor de $\mathrm{p}<0,05$ como estatisticamente significativo.

Os procedimentos e condutas foram aprovados pelo Comitê de Ética em Pesquisa da Unidade (CAAE 0052.0.228.00-09), além de indexado em www.clinicaltrials.gov como NCT01217164.

\section{Resultados}

O grupo 1 foi formado por 48 RN AIG (88,8\%) e 6 PIG (11,2\%), e o grupo 2 por 59 AIG (92,2\%), 1 PIG (1,0\%) e 4 GIG (6,8\%). O fluxograma de randomização dos participantes dos estudo é mostrado na figura 1 .

A dieta predominante em ambos os grupos foi o

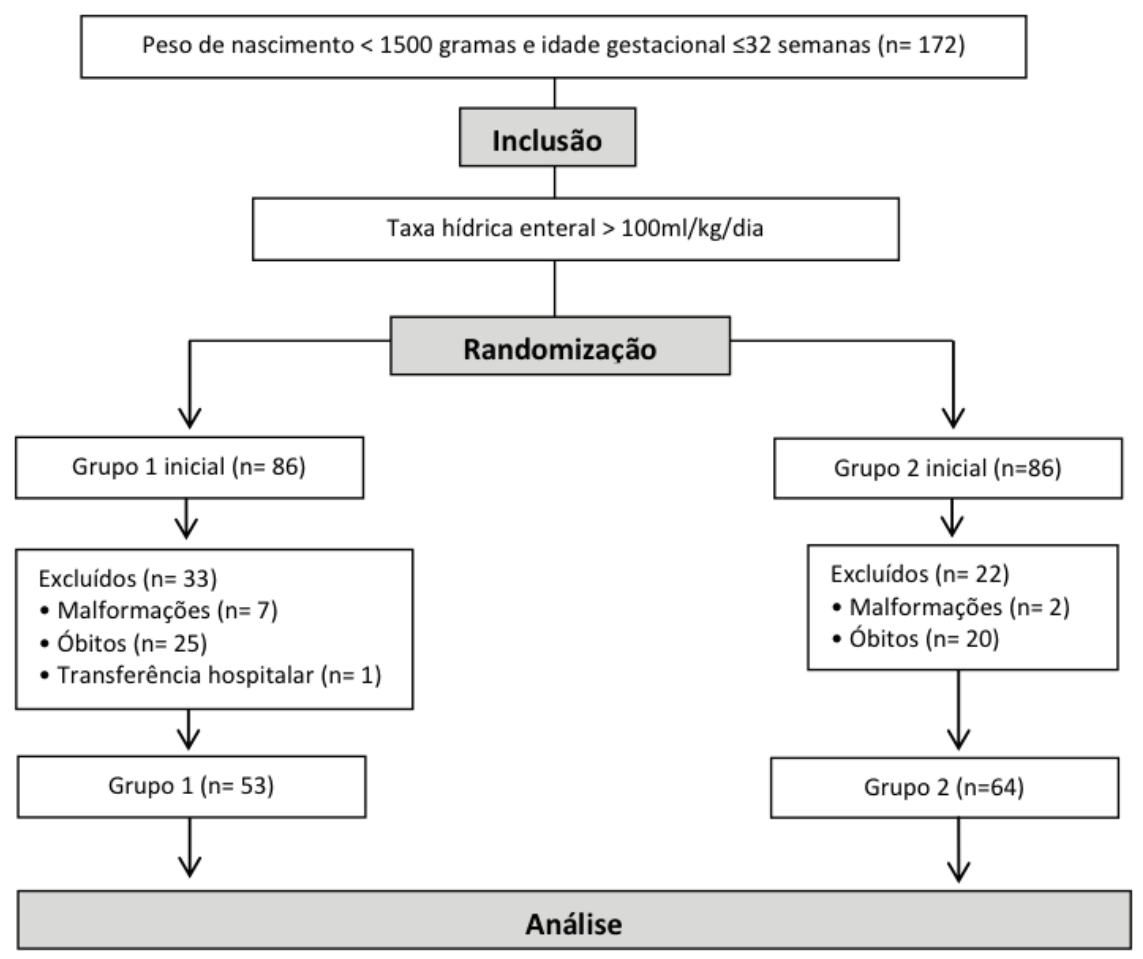

Figura 1. Fluxograma de randomização dos participantes do estudo. 
Eduardo M. Soares • Nutrição hiperproteica em recém-nascidos com muito baixo peso e evolução de índices antropométricos até a alta hospitalar: ensaio clínico randomizado

Tabela 1. Protocolo de nutrição dos grupos 1, com aporte proteico enteral diário de 4,5 gramas $/ \mathrm{kg} / \mathrm{dia}$, e 2, com aporte proteico enteral diário de 3,5 gramas $/ \mathrm{kg} /$ dia.

Dia 1: início de nutrição parenteral total com 3,0g/ $\mathrm{kg} /$ dia de aminoácidos e, se possível, dieta enteral em bolus com $20 \mathrm{ml} / \mathrm{kg} /$ dia. Complementação calórica com glicose (TIG 6,0-12,0mg/kg/min) em paralelo à nutrição parenteral total.

Dia 2: introdução de lipídeos (1,0g/ $/ \mathrm{gg} / \mathrm{dia})$, mantendo-se a dose de aminoácidos e a taxa de infusão de glicose na nutrição parenteral total. Progressão da dieta enteral em $20 \mathrm{ml} / \mathrm{kg} / \mathrm{dia}$.

Dia 3: aumento de lipídeos na nutrição parenteral total até a dose máxima (3,0g/kg/dia), mantendo-se taxa de infusão de glicose e proteína, continuando progressão de dieta.

Dia 4 dia 6: progressão da dieta e redução da infusão da nutrição parenteral total. Suspensão total da nutrição parenteral total quando a taxa hídrica enteral estabilizava em $100 \mathrm{ml} / \mathrm{kg} / \mathrm{dia}$.

\begin{tabular}{cc}
\hline \multicolumn{2}{c}{ Randomização } \\
\hline \multicolumn{1}{c}{ Grupo 1} \\
$\begin{array}{ll}\text { Dia } 7 \text { em diante: progressão da dieta até aporte } \\
\text { proteico enteral de 4,5g/kg/dia. }\end{array}$ & $\begin{array}{l}\text { Dia } 7 \text { em diante: progressão da dieta até aporte } \\
\text { proteico enteral de 3,5g/kg/dia. }\end{array}$ \\
\hline
\end{tabular}

TIG: taxa de infusão de glicose.

Tabela 2. Características demográficas e antropométricas expressas em média e desvio padrão ao início do estudo.

\begin{tabular}{l|c|c|c}
\hline & Grupo $\mathbf{1}(\mathbf{n}=\mathbf{5 3})$ & Grupo $\mathbf{2}(\mathbf{n}=\mathbf{6 4})$ & P-valor \\
\hline IG nascimento & $29,7 \pm 1,8$ semanas & $28,6 \pm 2,0$ semanas & 0,003 \\
\hline PN & $1100,5 \pm 251,1 \mathrm{~g}$ & $1034,5 \pm 250,1 \mathrm{~g}$ & 0,15 \\
\hline CN & $36,6 \pm 3,3 \mathrm{~cm}$ & $36,0 \pm 3,0 \mathrm{~cm}$ & 0,29 \\
\hline PCN & $26,6 \pm 1,9 \mathrm{~cm}$ & $25,6 \pm 1,9 \mathrm{~cm}$ & 0,006 \\
\hline Escore-Z PN & $-0,76 \pm 0,90$ & $-0,42 \pm 1,14$ & 0,09 \\
\hline Escore-Z CN & $-0,77 \pm 1,27$ & $-0,32 \pm 1,24$ & 0,05 \\
\hline Escore-Z PCN & $-0,26 \pm 1,18$ & $-0,16 \pm 1,26$ & 0,68 \\
\hline
\end{tabular}

IG: idade gestacional; PN: peso de nascimento; CN: comprimento de nascimento; PCN: perímetro cefálico de nascimento.

Tabela 3. Características demográficas e antropométricas expressas em média e desvio padrão na alta hospitalar.

\begin{tabular}{l|c|c|c}
\hline & Grupo $\mathbf{1}(\mathbf{n}=\mathbf{5 3})$ & Grupo $\mathbf{2}(\mathbf{n}=\mathbf{6 4})$ & P-valor \\
\hline IG corrigida na alta & $38,4 \pm 2,4$ semanas & $38,5 \pm 2,7$ semanas & 0,85 \\
\hline PA & $2677,5 \pm 716,5 \mathrm{~g}$ & $2583,6 \pm 518,7 \mathrm{~g}$ & 0,41 \\
\hline CA & $45,7 \pm 4,2 \mathrm{~cm}$ & $45,1 \pm 2,9 \mathrm{~cm}$ & 0,41 \\
\hline PCA & $33,4 \pm 1,7 \mathrm{~cm}$ & $33,1 \pm 1,6 \mathrm{~cm}$ & 0,31 \\
\hline Escore-Z PA & $-1,27 \pm 1,65$ & $-1,44 \pm 1,48$ & 0,64 \\
\hline Escore-Z CA & $-1,57 \pm 1,84$ & $-1,77 \pm 1,45$ & 0,55 \\
\hline Escore-Z PCA & $-0,37 \pm 1,38$ & $-0,57 \pm 1,41$ & 0,46 \\
\hline Variação escore-Z PN & $-0,52 \pm 1,23$ & $-1,02 \pm 1,10$ & 0,03 \\
\hline Variação escore-Z CN & $-0,79 \pm 1,55$ & $-1,45 \pm 1,44$ & 0,02 \\
\hline Variação escore-Z PCN & $-0,10 \pm 1,51$ & $-0,40 \pm 1,49$ & 0,30 \\
\hline Tempo de internação & $60,6 \pm 23,2$ dias & $68,7 \pm 25,4$ dias & 0,08 \\
\hline
\end{tabular}

IG: idade gestacional; PA: peso de alta; CA: comprimento de alta; PCA: perímetro cefálico de alta; PN: peso de nascimento; CN comprimento de nascimento; PCN: perímetro cefálico de nascimento. 


\section{Artigo original}

de fórmula láctea específica para prematuros. Além disso não houve diferenças no tempo de início de dieta em ambos os grupos e de taxa hídrica enteral máxima administrada durante a internação hospitalar.

As principais características demográficas e antropométricas na internação e na alta são descritas nas tabelas 2 e 3. Não houve diferenças estatisticamente significativas nas incidências de NEC ( $\mathrm{p}=0,70, \mathrm{OR}: 0,88$ ), deficit ponderal na alta ( $\mathrm{p}=0,27, \mathrm{OR}: 0,70)$, restrição de crescimento na alta $(\mathrm{p}=0,39$, OR: 0,82$)$ e deficit de PC na alta ( $\mathrm{p}=0,45$, OR: 0,67$)$.

\section{Discussão}

O presente ensaio clínico foi elaborado para avaliar as medidas antropométricas após a administração de nutrição hiperproteica em um grupo de RNMBP com IG $\leq 32$ semanas no período intra-hospitalar.

A nutrição pós natal é importante para o recém-nascido uma vez que vai determinar o seu crescimento e desenvolvimento futuros. ${ }^{17,18} \mathrm{O}$ fornecimento de proteínas feito pela gestante no período intraútero assegura o desenvolvimento de estruturas cerebrais e funções cognitivas que ocorrem ao final da gestação. ${ }^{8}$ Condições que levem à prematuridade (maternas ou fetais) fazem com que tal desenvolvimento seja abruptamente interrompido. ${ }^{18}$

O crescimento intrauterino fornece mais de 4,0g/ $\mathrm{kg} /$ dia de proteínas para o feto quando este chega a $24 \mathrm{a}$ 30 semanas de gestação, e 2,0-3,0g/kg/dia quando chega ao termo. ${ }^{6,8}$ Quando os bebês prematuros estão estáveis clinicamente, ocorre a transição da dieta parenteral para enteral.Poucos estudos são disponíveis em relação à nutrição enteral do paciente prematuro estável. É consenso que a melhor dieta para tais pacientes é o leite humano (LH), haja vista suas propriedades de proteção imunológica, de estímulo ao neurodesenvolvimento futuro e como fator protetor contra sepse neonatal tardia e NEC. ${ }^{19,20}$ Apesar de todos seus benefícios, o LH, quando oferecido ao prematuro, deve ser suplementado com proteínas e outros nutrientes, já que se estima que o deficit proteico gerado fique em torno de 0,6-0,8g/ $\mathrm{kg} /$ dia. $^{8}$

Os grupos formados demonstraram-se heterogêneos entre si, tanto em relação ao tamanho da amostra como em divisão pelo sexo, PCN, classificação de peso em relação à adequação para IG e idade gestacional de nascimento (IGN). Apesar disso, a proporcionalidade de participantes AIG (88,8\% versus $92,2 \%$, respectivamente para os grupos $1 \mathrm{e} 2$ ) foi a mesma entre os grupos, logo, a influência de um crescimento intrauterino restrito na curva de crescimento dos grupos fica menos provável, assim como as diferenças biológicas entre participantes.

As IGN diferiram entre si em 1,1 semana (29,7 versus 28,6 semanas, respectivamente, para grupo 1 e grupo 2). Apesar da DUM ter sido utilizada como principal método de avaliação em ambos os grupos $(67,9 \%$ versus $65,6 \%$, respectivamente, para grupo 1 e grupo 2 ), a diferença encontrada de IGN entre os grupos pode ter sido gerada por vieses inerentes à avaliação pelo USG e New Ballard Score. ${ }^{21}$ Quando analisados os PN e CN, e seus respectivos escores-Z, não foram encontradas diferenças significativas, o que demonstra a presença de homogeneidade dentre os diversos estratos analisados. É digno de nota que, em relação às médias de $\mathrm{PN}$, não houve desvio em relação à classificação da OMS de RNMBP.

Durante o período de internação hospitalar não foi observada diferença de tempo na introdução de dieta enteral $(p=0,58)$, sendo que em ambos os grupos predominou o uso do leite artificial. A TH enteral máxima administrada aos participantes não diferiu $(\mathrm{p}=0,07)$, assim como sua principal intercorrência esperada, NEC $(\mathrm{p}=0,70)$. Dejhalla ${ }^{22}$ apresenta resultados semelhantes de incidência de NEC(10\%) nos participantes submetidos à dieta hiperproteica. Há divergência com a literatura ${ }^{23,24}$ em relação ao citado, já que o excesso de TH administrada, em especial pela via enteral, predispõe à NEC. Devido à etiologia multifatorial e até momento obscura de tal doença, outros fatores adversos ao presente estudo provavelmente influenciaram em tal desfecho, como, por exemplo, a retirada precoce de antibióticos, sendo necessários mais estudos para elucidar tal correlação.

Nota-se que as variações de escore-Z tanto de PN quanto $\mathrm{CN}$ foram menores no grupo 1.É digno de nota que em nenhum dos dados analisados houve ganho, mas sim decréscimo. Durante o tempo de internação hospitalar não houve aceleração de velocidade de crescimento, mas sim desaceleração, apontando que $o$ CEUR é uma regra aos RNMBP, assim como suas consequências futuras. ${ }^{2,5,9,25}$ Van der Schoor ${ }^{26}$ encontrou que o balanço do aminoácido lisina era significativamente aumentado nos prematuros em dieta enteral plena e Dejhalla $^{22}$ conclui que a taxa de crescimento em seu estudo foi mais acentuada durante o período de dieta enteral exclusiva. Tal desaceleração menos acentuada é indicativa de possível prevenção de CEUR nos participantes expostos ao maior aporte proteico enteral durante internação hospitalar, apesar da pequena diferença de infusão proteica entre os grupos $(1,0 \mathrm{~g} / \mathrm{kg} / \mathrm{dia})$.

Não foram analisados, ao longo do trabalho, indicadores bioquímicos, tais como ureia, nitrogênio 
ureico, retinol ou albumina, como também a relação proteico-calórica administrada ou a relação de crescimento com ingesta calórica. $\mathrm{Hay}^{8}$ refuta a necessidade de acompanhamento de tais indicadores, principalmente aqueles relacionados ao metabolismo proteico. O principal motivo de alarde em relação à aporte hiperproteico enteral deriva do estudo de Goldman, ${ }^{10}$ que encontrou mais malefícios do que benefícios com a conduta. Thureen ${ }^{17}$ afirma que tais preocupações são infundadas, visto que a dieta oferecida por Goldman era de baixa qualidade quando comparada às atualmente disponíveis. Além disso, a produção de ureia é muito alta quando há altos índices de oxidação de aminoácidos: o excesso de aminoácidos não incorporado ao balanço basal proteico do prematuro é oxidado para produção energética. ${ }^{8,18}$ De fato, os aminoácidos são o segundo substrato mais utilizado por essa população, somente abaixo da glicose. Logo, é de se esperar que bebês prematuros mantenham tal padrão metabólico, esperando-se encontrar rotineiramente níveis mais elevados de ureia nesta população, não existindo evidência crível de que tais níveis sejam deletérios. ${ }^{8}$

Questiona-se a necessidade do acompanhamento da relação proteico-calórica e a incorporação proteica basal do prematuro, ${ }^{8,12,22}$ haja visto que quando se recebe aporte calórico de gordura e carboidratos abaixo de $60 \mathrm{kcal} / \mathrm{kg} /$ dia o organismo utiliza-se do catabolismo proteico para suprir a demanda energética mínima do organismo. ${ }^{27}$ Dessa forma, quando oferecidos mais que $60 \mathrm{kcal} / \mathrm{kg} / \mathrm{dia}$, todo o substrato proteico oferecido é utilizado para demandas nobres do organismo, como formação de massa magra e crescimento cerebral. Mesmo assim de nada adiantaria oferecer dieta hipercalórica além desses índices com o intuito de otimizar esse crescimento, pois a partir de $60 \mathrm{kcal} / \mathrm{kg} / \mathrm{dia}$ tudo o que é oferecido além acaba se resumindo à lipogênese. ${ }^{8}$

Não se pode concluir que as alterações metabólicas e antropométricas ocorridas no período de internação hospitalar permanecerão, nem mesmo que trarão benefícios a longo prazo. Contudo, reconhecer que houve diferença de crescimento entre os dois grupos, notadamente em peso e comprimento traz a necessidade de acompanhamento desses participantes, principalmente no que concerne ao desenvolvimento neurocognitivo e transtornos metabólicos de interesse. ${ }^{11,12}$

Por fim, cria-se assim o grande dilema do neonatologista: promover o crescimento normal do prematuro, mimetizando aquele encontrado no ambiente intrauterino, ${ }^{28}$ teoricamente associado com melhor desfecho neuromotor futuro e possivelmente associado à síndro- me metabólica ${ }^{11,12}$ ou manter a "subnutrição benéfica", ${ }^{17}$ com desfechos neuromotores deficitários ${ }^{1,2}$ e ausência de transtornos metabólicos futuros? Singhal, ${ }^{29}$ propõe diretamente que "[...] de forma equilibrada, a conduta corrente é promover aceleração de crescimento com aumento de aporte nutricional [...]considerando os efeitos do programming de crescimento pós-natal acelerado em tais bebês, focando na fisiopatologia basal e impacto clínico".

\section{Referências}

1. Hack M, Breslau N, Weissman B, et al. Effect of very low birth weight and subnormal head size on cognitive abilities at school age. N Engl J Med. 1991;325(4):231-7. http://doi.org/10.1056/ NEJM199107253250403

2. Franz AR, Pohlandt F, Bode $\mathrm{H}$, et al. Intrauterine, early neonatal, and postdischarge growth and neurodevelopmental outcome at 5.4 years in extremely preterm infants after intensive neonatal nutritional support. Pediatrics. 2009;123(1):e101-9. http://doi.org/10.1542/peds.2008-1352

3. Hay WW. Early postnatal nutritional requirements of the very preterm infant based on a presentation at the NICHD-AAP workshop on research in neonatology. J Perinatol . 2006;26 Suppl 2:S13-8. http://doi.org/10.1038/sj.jp.7211426

4. McLeod G, Sherriff J. Preventing postnatal growth failure--the significance of feeding when the preterm infant is clinically stable. Early Hum Dev. 2007;83(10):659-65. http://doi. org/10.1016/j.earlhumdev.2007.07.010

5. Thureen PJ. Early aggressive nutrition in the neonate. Pediatr Rev Am Acad Pediatr. 1999;20(9):e45-55.

6. Carlson SJ, Ziegler EE. Nutrient intakes and growth of very low birth weight infants. J Perinatol Off J Calif Perinat Assoc. 1998;18(4):252-8.

7. Ziegler EE, O'Donnell AM, Nelson SE, et al. Body composition of the reference fetus. Growth. 1976;40(4):329-41.

8. Hay WW, Thureen P. Protein for preterm infants: how much is needed? How much is enough? How much is too much? Pediatr Neonatol. 2010;51(4):198-207. http://doi.org/10.1016/ S1875-9572(10)60039-3

9. Embleton NE, Pang N, Cooke RJ. Postnatal malnutrition and growth retardation: an inevitable consequence of current recommendations in preterm infants? Pediatrics. 2001;107(2):270-3.

10. Goldman HI, Goldman J, Kaufman I, et al. Late effects of early dietary protein intake on low-birth-weight infants. J Pediatr. 1974;85(6):764-9.

11. Singhal A, Lucas A. Early origins of cardiovascular disease: is there a unifying hypothesis? Lancet . 2004;363(9421):1642-5. http://doi.org/10.1016/S0140-6736(04)16210-7

12. Ong KK, Ahmed ML, Emmett PM, et al. Association between postnatal catch-up growth and obesity in childhood: prospective cohort study. BMJ. 2000;320(7240):967-71.

13. Gibson RS. Principles of Nutritional Assessment. Oxford University Press; 2005.

14. Ballard JL, Khoury JC, Wedig K, et al. New Ballard Score, expanded to include extremely premature infants. J Pediatr. 1991;119(3):417-23.

15. Fenton TR, Kim JH. A systematic review and meta-analysis to revise the Fenton growth chart for preterm infants. BMC Pediatr. 2013;13:59. http://doi.org/10.1186/1471-2431-13-59 


\section{Artigo original}

16. Bell MJ, Ternberg JL, Feigin RD, et al. Neonatal necrotizing enterocolitis. Therapeutic decisions based upon clinical staging. Ann Surg. 1978;187(1):1-7.

17. Thureen PJ. The neonatologist's dilemma: catch-up growth or beneficial undernutrition in very low birth weight infants-what are optimal growth rates? J PediatrGastroenterolNutr. 2007;45 Suppl 3:S152-4. http://doi.org/10.1097/01. mpg.0000302962.08794.62

18. Vlaardingerbroek $\mathrm{H}$, van Goudoever JB, van den Akker CHP. Initial nutritional management of the preterm infant. Early Hum Dev. 2009;85(11):691-5. http://doi.org/10.1016/j.earlhumdev.2009.08.052

19. Simmer K. Aggressive nutrition for preterm infants--benefits and risks. Early Hum Dev. 2007;83(10):631-4. http://doi. org/10.1016/j.earlhumdev.2007.07.013

20. World Health Organization (WHO). WHO Global Database on Child Growth and Malnutrition [Internet]. WHO. [citado 14 de março de 2016]. Disponível em: http://www.who.int/nutgrowthdb/en/

21. Smith VC. Recém-nascido de alto risco: antecipação, avaliação, tratamento e desfechos.In: Cloherty JP, Eichenwald EC, Stark AR. Manual de Neonatologia. 7ed. Rio de Janeiro: Guanabara Koogan, 2015. p 59-71

22. Dejhalla M, Lahage N, Parvez B, et al. Early Postnatal Growth in a Subset of Convalescing Extremely-Low-Birth-Weight
Neonates: Approximating the "Index Fetus" Ex Utero. J Pediatr Gastroenterol Nutr. 2015;61(3):361-6. http://doi.org/10.1097/ MPG.0000000000000750

23. Lim JC, Golden JM, Ford HR. Pathogenesis of neonatal necrotizing enterocolitis. Pediatr Surg Int. 2015;31(6):509-18. http://doi.org/10.1007/s00383-015-3697-9

24. Caplan M. Neonatal necrotizing enterocolitis. In: Martin RJ, Fanaroff AA, Walsh M. Fanaroff \& Martin's Neonatal-Perinatal Medicine - Diseases of the Fetus and Infant. 10th edition. Philadelphia. Elsevier Saunders; 2015. p. 1423-32

25. Hack M, Fanaroff AA. Outcomes of children of extremely low birthweight and gestational age in the 1990's. Early Hum Dev. 1999;53(3):193-218.

26. Van der Schoor SRD, Reeds PJ, Stellaard F, et al. Lysine kinetics in preterm infants: the importance of enteral feeding. Gut. 2004;53(1):38-43.

27. Thureen PJ, Hay WW. Intravenous nutrition and postnatal growth of the micropremie. Clin Perinatol. 2000;27(1):197-219.

28. Aapcon 1998 Nutritional needs of preterm infants. In: Kleinman RE (ed) Pediatric Nutrition Handbook. American Academy of Pediatrics, Elk Groove Village; 1998. p. 55-79.

29. Singhal A. [Internet]. Should We Promote Catch-Up Growth or Growth Acceleration in Low-Birthweight Infants?. Vol. 81. Karger Publishers; 2015 [citado 14 de março de 2016]. Disponível em: http://www.karger.com/Article/Abstract/365803 